\title{
The Database of Galactic PNe in Innsbruck
}

From the DeNIS Sub-Database to a General Tool

\section{S. Kimeswenger, C. Kienel and H. Wildauer}

Institute of Astronomy, University of Innsbruck

Information in Astronomy is available in multiple form. There are the periodicals as well as printed catalogues. Electronic abstract and paper services as well as general data centers as provided by NED, CDS, NASA/ADC, STScI, ...... and give access via modern communication media. The Deep Near Infrared Southern Sky Survey consortium (DeNIS, Epchtein et al. Astrophys. and Space Sci., 217, 3) itself provides all the survey data at two dedicated centers. Regarding all these services we have to ask:

Why do we need a specialised PNe data base ?

During the last decade the available amount of data in all fields of astronomy has drastically expanded. The new digital sky surveys will extend that by at least another order of magnitude. The field of PNe research comprises about 1 percent of the total amount of publications in astronomy (Saurer \& Weinberger, 1994, Scientrometrics, 31, 85). However about 250 scientists publish about 200 papers (out of approximately 20.000 in total) per year in this field of science. Specialised electronic data bases being able to combine information and data from different authors will be increasingly important. The possibility to combine information and use it for selection criteria (e.g. color indices, line ratios, ...) makes it a powerful tool in addition to the great work already done for catalogues (Acker et al 1992, Strasbourg-ESO Catalogue of Galactic Planetary Nebulae, ESO Munich, Germany).

The DeNIS survey consortium has decided to set up - in addition to the servers containing all data without any cross correlation with other information - specialised data bases. We started to set up a data base on galactic PNe, accessable via world wide web (http://ast2.uibk.ac.at/). We plan, dependent on the collaboration with the worldwide community, to extend this information far above pure DeNIS-related matters. As a first step we implemented the coordinates of a huge set of objects once claimed to be a PN and added data from work done in Innsbruck. Narrow band images provided by Balick (1987, AJ, 94, $671)$ and finding charts from the STScI Digitized Sky Survey are already available. The images by Schwarz et al. $(1992, A \& A S, 96,23)$ will be available soon. An area for abstracts of papers submitted and an area for preprints for papers accepted is able to accept contributions from everywhere. The first DeNIS images about known PNe were already reduced and will be prepared for the database soon.

To be able to make this data base a common tool we take the opportunity to use this abstract also as a call for contributions. We invite anyone for running a test session and are waiting for your suggestions. Thank you !

Acknowledgements: The authors thank to R. Weinberger for valuable discussions and the other members of the DeNIS consortium (see e.g. http://www-denis.iap.fr or http://www.strw.leidenuniv.nl/denis) for their work. This work was partly carried out with support of the FWF project P10036-PHY. 